\title{
Self-Similarly Evolving and Minimally Dissipated Stable States of Plasmas Realized after Relaxation and Self-Organization Processes
}

\author{
Yoshiomi KONDOH, Toru HAKOIWA, Akihito OKADA, \\ Naohiro KOBAYASHI and Toshiki TAKAHASHI \\ Department of Electronic Engineering, Gunma University, Kiryu 376-8515, Japan
}

(Received 8 June 2006 / Accepted 31 August 2006)

\begin{abstract}
A novel set of simultaneous eigenvalue equations having dissipative terms are derived to find self-similarly evolving and minimally dissipated stable states of plasmas realized after relaxation and self-organization processes. By numerically solving the set of eigenvalue equations in a cylindrical model, typical spatial profiles of plasma parameters, electric and magnetic fields and diffusion factors are presented, all of which determine self-consistently with each other by physical laws and mutual relations among them, just as in experimental plasmas.
\end{abstract}

(c) 2006 The Japan Society of Plasma Science and Nuclear Fusion Research

Keywords: self-organization, self-similarly evolving state, minimally dissipative state, shear flow

DOI: $10.1585 /$ pfr. 1.043

A recent generalized theory of self-organization for finding eigenvalue equations to obtain self-similarly evolving and minimally dissipated stable states [1-6] has been shown to incorporate a previous theory [7] for obtaining the minimum dissipative state of magnetic energy, by means of which "the so-called Taylor state" [8] can be derived. The concept of selective decay together with that of helicity invariance in the traditional theories [8-10] is analytically proved in [3-5] to have theoretically unrelated with "relaxed states". The fusion plasma is also known to be described for simulations by a set of charge, mass, momentum, and energy conservation laws, and Maxwell's equations to follow its dynamic evolution and to analyze relaxation processes and relaxed states. However, replacement of any element in the set by "the so-called helicity conservation law" [8-10] makes the dynamic evolution untraceable due to this nonphysical law. Thus, it has been clarified that the all theoretical basis of the traditional theories has no theoretical and physical connection with simulation results $[11,12]$ and experimental ones $[13,14]$ of the relaxed plasma observed and reported so far. The generalized theory is also applicable to other relaxed states, such as non-Taylor states with non-uniform resistivity [12], and to dissipative dynamical structures, such as the soliton solutions of the viscid Korteweg-de Vries equation [15] and the vortex solutions of the two-dimensional incompressible viscous fluid equations $[6,16]$. The generalized theory is a universal theory unifying apparently different theories, such as those of minimum dissipation of magnetic energy in [7] and of minimal (enstrophy/energy) in [17], as analytically proved in [6].

In this paper, we present a novel set of simultane- ous eigenvalue equations to find self-similarly evolving and minimally dissipated stable states realized after relaxation and self-organization processes. We show numerically solved spatial profiles of plasma parameters, magnetic and electric fields and diffusion factors of electrical conductivity $\sigma$, viscosity $v$, and thermal conductivity $\kappa$, all of which determine self-consistently with each other by physical laws and mutual relations, just as in experimental plasmas $[13,14]$ and in simulation results $[11,12]$.

We have applied the generalized theory to the twofluid model for fully ionized, compressible, resistive, viscid fusion plasmas. Substituting all equations of mass, momentum and energy conservation laws for electrons and ions and Maxwell's equations with the displacement current neglected into the final equation of Eq. (13) in [6] meaning the self-similarly evolving and minimally dissipated stable states derived by the generalized theory, and taking account of negligibly small mass of electron, quasineutrality, negligibly small ion thermal conductivity, negligible electron momentum and viscosity, we obtain the following set of simultaneous eigenvalue equations;

$$
\begin{aligned}
& \frac{\partial\left(m_{\mathrm{i}} n_{\mathrm{i}}\right)}{\partial t}=-\nabla \cdot\left(m_{\mathrm{i}} n_{\mathrm{i}} \boldsymbol{u}_{\mathrm{i}}\right)=-\lambda_{\mathrm{i} 1} m_{\mathrm{i}} n_{\mathrm{i}}, \\
& \frac{\partial\left(m_{\mathrm{i}} n_{\mathrm{i}} \boldsymbol{u}\right)}{\partial t}=-\nabla p-\nabla \cdot \boldsymbol{\Pi}_{\mathrm{i}}-m_{\mathrm{i}} n_{\mathrm{i}}(\boldsymbol{u} \cdot \nabla) \boldsymbol{u}+\boldsymbol{j} \times \boldsymbol{B} \\
&=-Z \lambda_{\mathrm{im}} m_{\mathrm{i}} n_{\mathrm{i}} \boldsymbol{u}, \\
& \frac{\partial \boldsymbol{B}}{\partial t}=-\nabla \times\left\{\frac{1}{\mu_{0}}\left[\sigma_{/ /}^{-1}(\nabla \times \boldsymbol{B})_{/ /}+\sigma_{\perp}^{-1}(\nabla \times \boldsymbol{B})_{\perp}\right)\right] \\
&\left.-\boldsymbol{u} \times \boldsymbol{B}+\frac{\boldsymbol{j} \times \boldsymbol{B}-\nabla p_{\mathrm{e}}}{e n_{\mathrm{e}}}\right\}=-\lambda_{B} \boldsymbol{B},
\end{aligned}
$$




$$
\begin{aligned}
\frac{\partial p}{\partial t}=- & \nabla(p \boldsymbol{u})+\left\{\nabla \cdot\left(\kappa_{\mathrm{e} / /} \nabla_{/ /} T_{\mathrm{e}}+\kappa_{\mathrm{e} \perp} \nabla_{\perp} T_{\mathrm{e}}\right)\right. \\
& +\eta_{/ / j_{/ /}^{2}}+\eta_{\perp} j_{\perp}^{2}-p \nabla \cdot \boldsymbol{u} \\
& \left.-\sum_{i, j=1}^{3} \boldsymbol{\Pi}_{\mathrm{i} i, j} \partial u_{\mathrm{i} i} / \partial x_{j}\right\}(\gamma-1)=-\lambda_{p} p,
\end{aligned}
$$

where $\lambda$ is eigenvalue normalized characteristic time scale for each quantity, and $\boldsymbol{\Pi}_{\mathrm{i}}$ is the stress tensor of ion. Using dominant terms in the relaxed quasi-steady state with divergent-free flows, definition of dissipative factors and conventional normalization by central values of quantities and others as shown below, we obtain the following normalized eigenvalue equations for the case of an axisymmetric system from Eqs. (1) - (4) ;

$$
\begin{aligned}
& \overline{\boldsymbol{u}} \cdot \bar{\nabla} \bar{n}=\Lambda_{\mathrm{im}}^{2} \bar{n}, \\
& \bar{\nabla} \times\left(\bar{\sigma}^{-1} \bar{\nabla} \times \overline{\boldsymbol{B}}_{\mathrm{p}}\right)=\Lambda_{B \mathrm{p}}^{2} \overline{\boldsymbol{B}}_{\mathrm{p}}, \\
& \bar{\nabla} \times\left(\bar{\sigma}^{-1} \bar{\nabla} \times \overline{\boldsymbol{B}}_{\mathrm{t}}\right)=\Lambda_{B \mathrm{t}}^{2} \overline{\boldsymbol{B}}_{\mathrm{t}}, \\
& \frac{1}{2} \bar{\nabla} \bar{p}+\bar{v} \bar{\nabla} \times \bar{\nabla} \times \overline{\boldsymbol{u}}+\bar{n}(\overline{\boldsymbol{u}} \cdot \bar{\nabla}) \overline{\boldsymbol{u}}-\overline{\boldsymbol{j}} \times \overline{\boldsymbol{B}}=\Lambda_{\mathrm{mu}}^{2} \bar{n} \overline{\boldsymbol{u}}, \\
& \bar{\nabla} \cdot\left(\bar{\kappa}_{\mathrm{e} \perp} \overline{\nabla_{\perp}} \bar{T}_{\mathrm{e}}\right)+\frac{6.81 \times 10^{-5} B_{0}^{4} \bar{\sigma}^{-1} \bar{j}^{2}}{\mu_{0}^{2} T_{0 \mathrm{e}}^{2} \alpha^{1 / 2} n_{0}^{2}}=-\Lambda_{\mathrm{Te}}^{2} \bar{n} \bar{T}_{\mathrm{e}},
\end{aligned}
$$

where the subscripts $\mathrm{t}$ and $\mathrm{p}$ denote toroidal and poloidal components, respectively, and normalization factors are embedded by multiplying them to corresponding eigenvalues. A relation of $T_{\mathrm{i}}=\alpha T_{\mathrm{e}}$ with a factor $\alpha$ is assumed to determine $T_{\mathrm{i}}$ because of degeneration of two fluid model to one fluid model. The normalization and normalized dissipative factors are

$\bar{t} \equiv t /\left(a / v_{\mathrm{A}}\right), v_{\mathrm{A}} \equiv B_{0} / \sqrt{\mu_{0} \rho_{0}}, \bar{\nabla} \equiv a \nabla, \bar{\rho}_{\mathrm{m}} \equiv \rho_{\mathrm{m}} / \rho_{0}$, $\bar{n} \equiv n / n_{0} \equiv n_{\mathrm{e}} / n_{0}, \overline{\boldsymbol{u}} \equiv \boldsymbol{u} / v_{\mathrm{A}}, \overline{\boldsymbol{B}} \equiv \boldsymbol{B} / \boldsymbol{B}_{0}, \overline{\boldsymbol{E}} \equiv \boldsymbol{E} /\left(v_{\mathrm{A}} B_{0}\right)$, $\overline{\boldsymbol{j}} \equiv \boldsymbol{j} /\left(B_{0} / \mu_{0} a\right), \bar{T}_{\mathrm{e}} \equiv T_{\mathrm{e}} / T_{0 \mathrm{e}}, \bar{\sigma} \equiv \bar{T}_{\mathrm{e}}^{\frac{3}{2}}, \bar{\kappa}_{\mathrm{e} \perp} \equiv \bar{n}^{2} \bar{T}_{\mathrm{e}}^{-\frac{1}{2}} / \bar{B}^{2}$, $\bar{v}_{0 \mathrm{i}} \equiv \bar{T}_{\mathrm{i}}^{\frac{5}{2}}\left(3.82 \times 10^{-6} T_{\mathrm{i} 0}^{\frac{5}{2}} \sqrt{\mu_{0}} / Z \ln \Lambda a B_{0} \sqrt{\rho_{0}}\right)$, and $T_{\mathrm{e}}[\mathrm{eV}]$.

In the limiting case of uniform $\sigma$ and negligible $v$ and $\kappa$, Eqs. (1) - (4) and/or Eqs. (5) - (9) lead to the so-called Taylor state, just the same as in [7]. In general cases, however, these equations can be applicable to finite beta confinement systems of the Tokamak, the reversed field pinch (RFP), the field reversed configuration (FRC), and so on. Using the cylindrical model for simplicity and the 4 rank and 4th order Runge Kutta method under suitable boundary conditions on measurable quantities by referring to experimental data [14], we have numerically solved Eqs. (5) (9) to get self-organized configurations of the RFP. A typical result is shown in Figs. 1 (a) and 1 (b) for a case of $\alpha=0.5$, where the radial electric field $E_{\mathrm{r}}$ mainly comes from the Hall term. It is seen from the data profiles that all physical quantities are related self-consistently with each other, i.e., $\kappa_{\mathrm{e} \perp}$ is determined by $n, T_{\mathrm{e}}$ and $B$, and $T_{\mathrm{e}}$ is determined by $\kappa_{\mathrm{e} \perp}, \sigma$ and $j$, and so on, to lead to negligibly
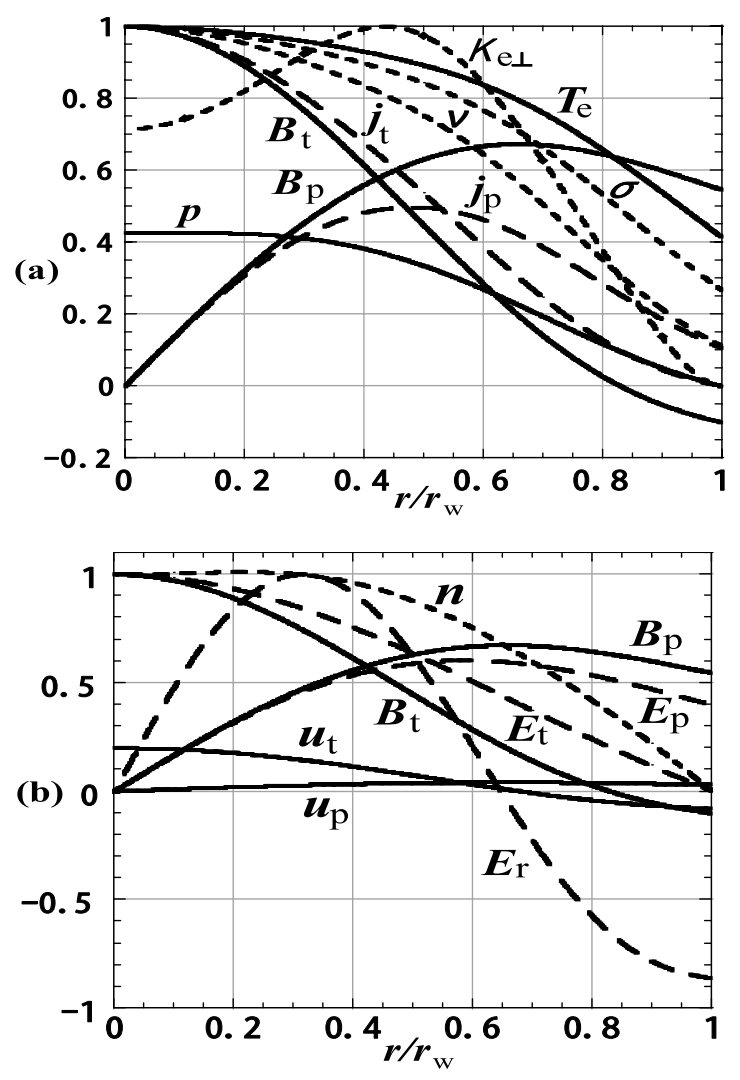

Fig. 1 A typical numerical result of the self-similarly evolving and minimally dissipated stable state for RFP plasmas.

small current density at the boundary wall like as in experimental plasmas. We also find from both profiles of $u_{\mathrm{p}}$ and $u_{\mathrm{t}}$ in Fig. 1 (b) that there exists the shear flow which depends on the profile of $v$, i.e., on that of $T_{\mathrm{i}}$, and would contribute to stabilization of the self-organized RFP plasma.

In conclusion, we have derived a novel set of simultaneous eigenvalue equations for finding self-similarly evolving and minimally dissipated stable states realized after relaxation and self-organization processes (cf. Eqs. (1) (4) or Eqs. (5) - (9)). The set of simultaneous equations is applicable to all types of magnetically confined fusion plasmas. Solving numerically the set of equations in the cylindrical model, we have shown typical self-organized configurations of the RFP plasma including a lot of spatial information on related physical quantities useful for detailed experimental investigation. It should be emphasized that all physical quantities of interest are self-consistently determined by physical laws and mutual relations among them.

[1] Y. Kondoh, Phys. Rev. E 48, 2975 (1993).

[2] Y. Kondoh, Phys. Rev. E 49, 5546 (1994).

[3] Y. Kondoh et al., J. Plasma Fusion Res. SERIES, 5, 598 (2002).

[4] Y. Kondoh et al., J. Plasma Fusion Res. SERIES 6, 601 (2004).

[5] Y. Kondoh et al., to be published in J. Plasma Physics 
(2006).

[6] Y. Kondoh et al., Phys. Rev. E 70, 066312-1 (2004).

[7] S. Chandrasekhar et al., Proc. Natl. Acad. Sci. 44, 285 (1958).

[8] J.B. Taylor, Phys. Rev. Lett. 33, 1139 (1974).

[9] L.C. Steinhauer and A. Ishida, Phys. Plasmas 4, 2609 (1998).

[10] Z. Yoshida and S.M. Mahajan, Phys. Rev. Lett. 88, 095001 (2002).
[11] R. Horiuchi and T. Sato, Phys. Rev. Lett. 55, 211 (1851).

[12] Y. Kondoh et al., J. Phys. Soc. Jpn. 63, 546 (1994).

[13] Kondoh et al., J. Phys. Soc. Jpn. 62, 2038 (1993).

[14] Y. Yagi et al., Nucl. Fusion 45, 138 (2005).

[15] Y. Kondoh and J.W. Van Dam, Phys. Rev. E 52, 1721 (1995).

[16] Y. Kondoh et al., Phys. Rev. E 54, 3017 (1996).

[17] W.H. Matthaeus et al., Phys. Rev. Lett. 66, 2731 (1991). 